\title{
Avaliação das condições periodontais e de higiene bucal em escolares com transtornos neuropsicomotores
}

\author{
Evaluation of periodontal conditions and oral hygiene in school children with neuropsychomotor disorders
}

\author{
Karen Silva NASILOSKI ${ }^{a}$, Ethieli Rodrigues da SILVEIRA , \\ João Batista CÉSAR NETO ${ }^{\text {b }}$ Lisandrea Rocha SCHARDOSIM ${ }^{\text {a* }}$
}

aFaculdade de Odontologia, UFPel - Universidade Federal de Pelotas, Pelotas, RS, Brasil

${ }^{b}$ Faculdade de Odontologia, USP - Universidade de São Paulo, São Paulo, SP, Brasil

\begin{abstract}
Resumo
Introdução: A doença cárie e as alterações periodontais são problemas frequentemente encontrados em pacientes com deficiência. Objetivo: Avaliar as condições periodontais e de higiene bucal em escolares com deficiência neuropsicomotora matriculados em um centro de reabilitação. Método: A pesquisa envolveu entrevista com os responsáveis e exame clínico do escolar, para registro do índice de placa e do índice de sangramento. Após o exame, foi realizada profilaxia profissional, orientação de higiene bucal e esclarecimento de dúvidas. Os dados foram observados por meio de estatística descritiva. Resultado: A amostra foi constituída por 41 escolares com deficiência neuropsicomotora, com idades entre sete e 14 anos, sendo 20 do sexo feminino (48,8\%) e 21 do sexo masculino $(51,2 \%)$, e com idade média de 10,8 anos. A maioria dos pacientes tinha a figura da mãe como principal cuidador (85\%). Observou-se que $71 \%$ dos escolares necessitavam de ajuda para realizar a higiene bucal e quase $40 \%$ dos cuidadores apresentavam dificuldade para realizá-la, principalmente pelo fato de o paciente "não abrir a boca". Quase a totalidade dos escolares não usava fio dental. Constatou-se, ainda, que 87,2\% apresentaram sangramento gengival à sondagem e que, em $84,7 \%$, verificou-se índice de placa maior ou igual a '2'. Conclusão: Concluiu-se que os escolares com deficiência neuropsicomotora incluídos neste estudo apresentaram condições periodontais e de higiene bucal insatisfatórias, demonstrando a necessidade de serem instituídas ações preventivas focadas na orientação aos pacientes e cuidadores, para a efetividade da higiene bucal.
\end{abstract}

Descritores: Higiene bucal; pessoas com deficiência; gengivite.

\begin{abstract}
Introduction: Caries and periodontal disease are problems frequently affecting patients with disabilities. Objective: In this study we aim to evaluate the periodontal conditions and the oral hygiene in students with neuropsychomotor disorders enrolled in a rehabilitation center. Method: Data collect was based in interview with the responsible and the scholar's clinical exam to register the plaque index and the bleeding index. After the clinical exam were performed professional prophylaxis, oral hygiene orientation and explanation of doubts. Result: The sample consisted of 41 students with neuropsychomotor disabilities, with ages among 7 and 14 years, 20 females (48.8\%) and 21 males (51.2\%) with a mean age of 10.8 years. Most patients had the figure of the mother as primary caregiver (85\%). It was observed that $71 \%$ of students need help in performing oral hygiene and nearly $40 \%$ of caregivers had difficulty doing it, in most of the cases because the patient "did not open his mouth". Almost the totality of the students doesn't use dental floss. It was also found that $87.2 \%$ had gingival bleeding and $84.7 \%$ had the plaque index greater or equal to 2. Conclusion: It was concluded that disabled students included in this study had bad periodontal conditions and poor oral hygiene. It shows the need of preventive programs to guide themselves and the caregivers, seeking for effectiveness of oral hygiene.
\end{abstract}

Descriptors: Oral hygiene; disabled persons; gingivitis.

\section{INTRODUÇÃO}

A doença cárie e as alterações periodontais são problemas frequentemente encontrados em pacientes com deficiência e a dificuldade em manter bons níveis de saúde bucal deve-se tanto ao comprometimento físico e/ou mental quanto às barreiras sociais que estes pacientes enfrentam ${ }^{1,2}$. Além disso, os antipsicóticos, antidepressivos tricíclicos e lítio, medicações amplamente prescritas para tratamentos psiquiátricos, têm notáveis efeitos anticolinérgicos, resultando em xerostomia crônica e maior risco para o desenvolvimento de cárie, gengivite, candidíase e lesões na mucosa bucal ${ }^{3,4}$. 
Apesar da necessidade de um acompanhamento odontológico adequado, a OMS (Organização Mundial da Saúde) relata que apenas $3 \%$ da população total de deficientes tem acesso aos tratamentos odontológicos. Corroborando com esta informação, estudos revelam que as crianças com comprometimento físico ou mental mais severo têm ainda menos acesso aos serviços odontológicos ${ }^{2,5}$. Isto ocorre devido à desinformação e à falta de comprometimento dos responsáveis, ao custo do tratamento, à presença de barreiras arquitetônicas, que dificultam o acesso aos serviços, e à ausência de capacitação profissional e grupos de estudo que discutam métodos facilitadores de prevenção e tratamento odontológico voltado para esses pacientes ${ }^{5-7}$.

Os programas de saúde bucal que são implementados para pacientes com deficiência visam à motivação para o controle mecânico efetivo da placa bacteriana. No entanto, quando estes pacientes apresentam alguma deficiência motora, podem tornar-se incapazes de realizar a higiene bucal sozinhos e os cuidadores devem assumir tal responsabilidade ${ }^{8}$. Segundo a literatura ${ }^{9,10}$, estes cuidadores têm atividades diárias acumuladas que, somadas com a ansiedade e a ideia pré-concebida de que cuidados odontológicos são impossíveis, podem levar ao adiamento ou mesmo ao esquecimento destas práticas. Agrava-se o fato de que, muitas vezes, profissionais de outras áreas, bem como escolas e centros de reabilitação, não estão atentos quanto à importância da saúde bucal e à necessidade de encaminhamento odontológico precoce ${ }^{4,11}$.

Estudos epidemiológicos e que avaliem as condições de saúde bucal desta clientela específica são fundamentais para sugerir estratégias preventivas e educativas adequadas. Dessa forma, o objetivo deste trabalho foi avaliar as condições periodontais e de higiene bucal em escolares de sete a 14 anos de idade, com distúrbios neuropsicomotores, matriculados em um centro de reabilitação.

\section{MÉTODO}

Foi realizado um estudo observacional do tipo transversal, envolvendo todos os escolares, entre sete e 14 anos de idade, matriculados no Centro de Reabilitação de Pelotas (CERENEPE), instituição filantrópica de referência no município, para a reabilitação de indivíduos com deficiência neuropsicomotora. O projeto de pesquisa foi previamente submetido ao Comitê de Ética em Pesquisa da Faculdade de Odontologia da Universidade Federal de Pelotas (UFPel) e aprovado para execução (Parecer n 38/07). Previamente ao início da coleta de dados, foi realizado um estudo piloto, a fim de adequar a ficha para coleta de dados, e um treinamento teórico e prático. O treinamento prático foi realizado pela examinadora e por um pesquisador considerado padrão ouro em dez indivíduos com deficiência neuropsicomotora da mesma faixa etária do grupo de estudo. Os resultados dos exames eram comparados e, havendo discordância, a discussão era proposta. Os escolares que participaram do treinamento prático não fizeram parte da amostra.

As informações foram coletadas através de entrevista com o responsável e exame clínico do paciente, os quais foram conduzidos no consultório odontológico da instituição filantrópica.

$\mathrm{Na}$ entrevista com o responsável, foram coletados dados socioeconômicos, demográficos, história médica e odontológica do escolar. Após esta etapa, o exame clínico foi realizado na cadeira odontológica, sob campo seco e iluminação artificial. Pacientes pouco colaboradores foram estabilizados na cadeira por meio de contenção física, realizada pelos cuidadores e pelo anotador. A higiene bucal e as condições periodontais foram avaliadas empregando-se o Índice de Placa ${ }^{12}$ e o Índice de Sangramento ${ }^{13}$, respectivamente. Para o exame, optou-se por utilizar os dentes índices empregados e descritos no Índice de Löe, Silness ${ }^{12}$, sendo examinados os dentes 16, 12, 24, 36, 32 e 44 de cada criança. A sequência de exame seguiu essa mesma ordem. Cada dente índice foi seco com um jato de ar, por aproximadamente dez segundos, e visualizado com auxílio de iluminação artificial, para identificar placa e sangramento gengival espontâneo. Após tal verificação, a margem gengival dos dentes foi contornada pelo toque suave da extremidade romba da sonda periodontal (cerca de 0,5 $\mathrm{mm}$ de profundidade), para que fosse obtido o escore do índice de placa de cada face. O maior escore encontrado no exame foi considerado para a classificação do paciente, para ambos os índices $\mathrm{O}$ escore de placa foi atribuído de acordo com o descrito por Löe, Silness ${ }^{12}$, considerando-se a espessura da placa na área gengival das faces dos dentes, não levando em consideração a extensão da placa na coroa dos dentes. O escore ' 0 ' é atribuído quando a área gengival do dente está livre de placa. No escore ' 1 ', a olho nu, não se observa placa in situ, mas pode-se observar placa na sonda, quando esta é passada no dente. Já no escore '2', a área gengival é coberta por uma camada de placa de espessura média, visível a olho nu. Por fim, no escore ' 3 ', há forte acúmulo de placa na área gengival e na face do dente.

Após o registro das informações advindas do exame clínico, foi realizada profilaxia profissional dos dentes, foram oferecidas orientações de higiene bucal aos pacientes e cuidadores, e entregue um kit de higiene, contendo escova, dentifrício fluoretado e fio dental. Os pacientes com necessidade de tratamento odontológico foram encaminhados ao Projeto de Extensão "Acolhendo Sorrisos Especiais”, da Faculdade de Odontologia da UFPel. Os dados e as frequências absolutas e relativas foram registrados em uma planilha eletrônica do Microsoft Excel versão 2010, e observados por estatística descritiva.

\section{RESULTADO}

Dos 50 escolares com deficiência neuropsicomotora, 41 foram incluídos. Nove não foram incluídos devido à ausência do Termo de Consentimento Livre e Esclarecido e/ou impossibilidade de realizar o exame clínico pela falta de colaboração do escolar. Em relação ao sexo, foram 20 pertencentes ao sexo feminino (48,8\%) e 21 , ao sexo masculino (51,2\%), tendo idade média de 10,8 anos. A maioria dos pacientes tinha a figura da mãe como principal cuidador (85\%). A distribuição da amostra de acordo com as características estudadas está apresentada nas Tabelas 1 e 2.

Em relação ao uso da medicação, verificou-se que $66,7 \%$ dos pacientes recebiam medicações de forma fracionada, sendo que 12 crianças faziam uso da medicação também durante a noite.

Os Índices de Placa e Sangramento, verificados em 39 escolares, estão dispostos nas Figuras 1 e 2, respectivamente. Em dois escolares, não foi realizado o exame clínico devido à impossibilidade de colaboração. 


\section{DISCUSSÃO}

Os pacientes com deficiência são considerados de risco para as doenças bucais ${ }^{1,2,14}$ e a Odontologia deve investir na busca de estratégias para identificar estes fatores e desenvolver medidas preventivas e educativas efetivas para minimizar a instalação das doenças.

Tabela 1. Distribuição dos escolares com distúrbios neuropsicomotores de acordo com as características estudadas $(n=41)$

\begin{tabular}{lcc}
\hline \multicolumn{1}{c}{ Características } & n & $\%$ \\
\hline Tipo de Deficiência & 41 & 100 \\
\hline Síndrome de Down & 17 & 41,5 \\
\hline Deficiência Intelectual & 14 & 34,1 \\
\hline Paralisia Cerebral & 07 & 17,1 \\
\hline Outros & 03 & 7,2 \\
\hline Medicação utilizada & 41 & 100 \\
\hline Nenhuma & 23 & 56,1 \\
\hline Anticonvulsivo & 07 & 38,8 \\
\hline Neuroléptico & 06 & 33,3 \\
\hline Antidepressivo & 02 & 11,1 \\
\hline Outros & 03 & 16,8 \\
\hline
\end{tabular}

Tabela 2. Distribuição dos escolares com distúrbios neuropsicomotores de acordo com os hábitos de higiene bucal $(n=41)$

\begin{tabular}{|lcc|}
\hline \multicolumn{1}{c}{ Características } & $\mathbf{n}$ & $\%$ \\
\hline Higiene bucal & 41 & 100 \\
\hline Sim & 38 & 95,1 \\
\hline Não & 03 & 4,9 \\
\hline Como é feita a higiene bucal & 41 & 100 \\
\hline Não é realizada & 3 & 7,3 \\
\hline Com ajuda do cuidador & 27 & 71 \\
\hline Sozinho & 11 & 29 \\
\hline Dificuldade para realizar a higiene bucal & 41 & 100 \\
\hline Sim & 16 & 39,1 \\
\hline Não & 25 & 60,9 \\
\hline Tipo de dificuldade para realizar a higiene & $16^{*}$ & 100 \\
\hline bucal & 13 & 81,3 \\
\hline "Não abre a boca" & 03 & 18,7 \\
\hline "Não deixa" & 41 & 100 \\
\hline Uso do fio dental & 03 & 7,3 \\
\hline Sim & 38 & 92,7 \\
\hline Não & & \\
\hline
\end{tabular}

*Incluídos apenas os que têm dificuldade para realizar a higiene bucal.
A grande maioria dos escolares incluídos neste estudo é dependente de um cuidador para realizar a higiene bucal, sendo este cuidador representado pela figura da mãe em mais de $85 \%$ dos casos. Resultado semelhante foi encontrado por Campanaro et al. ${ }^{10}$, em que $88 \%$ dos cuidadores eram as mães. Assim, instruir a família, especialmente as mães, sobre dieta e higiene bucal adequadas, benefícios do tratamento odontológico precoce e dos retornos periódicos preventivos deve ser a primeira medida para assegurar a saúde bucal destes pacientes ${ }^{15-17}$. É preciso que a família entenda a importância do monitoramento da saúde bucal no consultório odontológico e da manutenção do vínculo entre profissional, paciente e família ${ }^{7,15,16}$. Se os cuidados com a saúde bucal forem instituídos precocemente e tiverem suporte dos pais, a necessidade de tratamentos odontológicos mais complexos pode ser reduzida ${ }^{18}$.

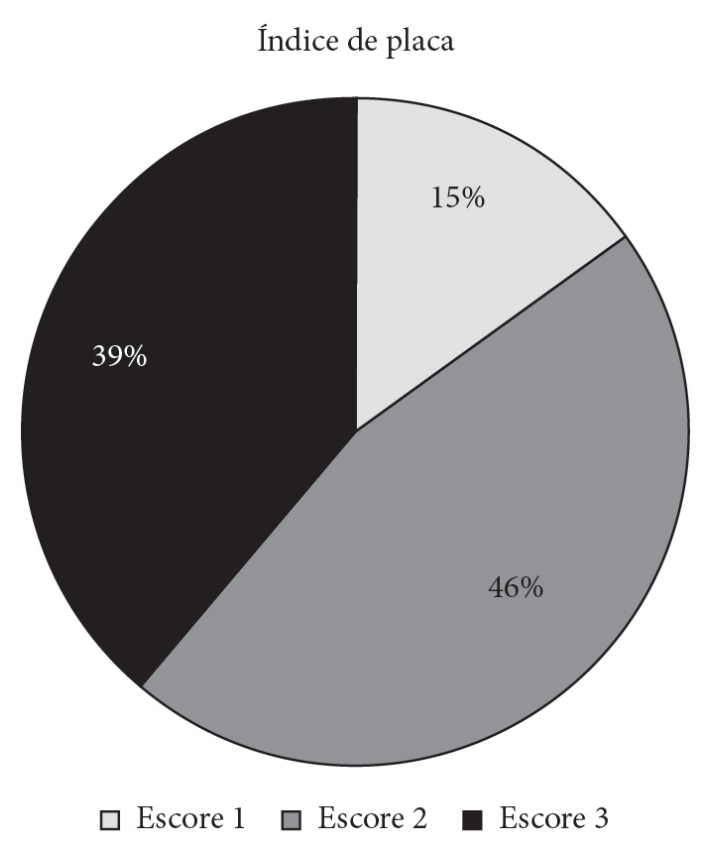

Figura 1. Distribuição dos escolares portadores de distúrbios neuropsicomotores de acordo com os escores do Índice de Placa $(n=39)$.

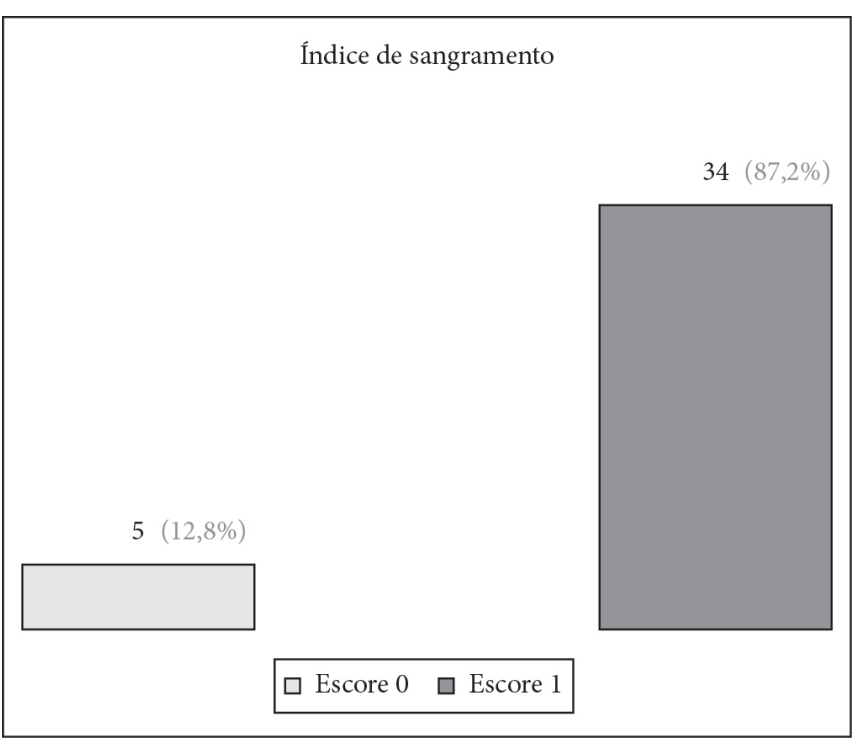

Figura 2. Distribuição dos escolares portadores de distúrbios neuropsicomotores de acordo com o Índice de Sangramento $(n=39)$. 
Assim como encontrado por Sacchetto et al. ${ }^{1}$, os resultados deste estudo demonstraram que o emprego de medicações de uso contínuo, tais como anticonvulsivos, neurolépticos e antidepressivos, faz parte da rotina de muitos pacientes com deficiência (44\%), o que pode acarretar alterações na cavidade bucal. A hiperplasia gengival, a xerostomia e a hipersalivação são exemplos destes efeitos adversos. Ademais, muitos destes fármacos apresentam sacarose em sua formulação, predispondo ao desenvolvimento da doença cárie ${ }^{3,15,19,20}$. Neste contexto, verifica-se a importância de orientar os cuidadores quanto às alterações bucais decorrentes da medicação, seus efeitos deletérios na dentição e a importância da escovação após sua administração $0^{15,21}$.

Em relação ao índice de placa, verificou-se que $85 \%$ dos pacientes apresentaram uma quantidade de placa madura representativa (Figura 1). Outros autores ${ }^{14,18,22,23}$ também verificaram condições de higiene bucal insatisfatórias quando empregaram o IHOS e enfatizaram a necessidade de atenção odontológica mais efetiva para esses indivíduos. Constatou-se, ainda, que $87,2 \%$ dos pacientes apresentaram sangramento gengival espontâneo (Figura 2), o que indica um alto risco para doença periodontal mais severa na idade adulta, corroborando com outros estudos realizados ${ }^{24,25}$.

A literatura relata índices preocupantes sobre a qualidade da higiene bucal desta população $0^{8,26}$. No trabalho conduzido por Campanaro et al. ${ }^{10}$, apenas $10 \%$ dos cuidadores referiram realizar sem problemas a higiene bucal das crianças com deficiência sob sua responsabilidade. Neste trabalho, dentre as dificuldades relatadas pelos cuidadores para a realização da higiene bucal, destacou-se a limitação da abertura de boca (81,3\%). O emprego de abridores de boca para uso domiciliar ainda é pouco divulgado e deve ser indicado para indivíduos com distúrbios neuropsicomotores pouco colaboradores ${ }^{27}$. Os abridores podem ser encontrados em diversos materiais, como monoblocos de borracha, metálicos e dedeiras ${ }^{27,28}$. Alguns autores ${ }^{6,29}$ ainda recomendam os abridores de boca fabricados manualmente, utilizando pet ou espátulas de madeira, visto seu baixo custo e praticidade. $\mathrm{O}$ uso destes instrumentos deve ser ampliado, considerando-se que, através da correta orientação, a família pode buscar a superação de dificuldades encontradas durante a higiene bucal de pacientes com deficiência.

Neste estudo, foi constatado que mais de $90 \%$ dos escolares com deficiência neuropsicomotora não utilizavam fio dental, corroborando com resultados encontrados em outros estudos semelhantes. Em Azrina et al. ${ }^{26}$, apenas sete dos 114 participantes utilizavam fio dental, assim como Sacchetto et al. ${ }^{1}$ verificaram que $70 \%$ dos pacientes referiram não possuir este hábito. O acesso do fio ao espaço interproximal pode ser facilitado com o uso do porta-fio dental, que consiste em duas hastes nas quais o fio é fixado. Seu uso é indicado para pacientes com deficiências motoras e deve ser estimulado com o intuito de facilitar esta etapa da higiene. Estudos demonstram que o porta-fio é eficaz, causa menos traumas aos tecidos gengivais e é mais aceito pelas crianças ${ }^{30}$.

As consequências da higiene bucal ineficiente de pessoas com deficiência não se limitam à cavidade bucal, visto que já está demonstrada a associação da higiene bucal inadequada com deterioração na saúde bucal e piora na qualidade de vida desta população ${ }^{31,32}$. Assim, todos estes dados evidenciam a importância de instituir programas de atenção à saúde bucal dirigidos aos pacientes com deficiência e suas famílias, a fim de promover ações preventivas efetivas, esclarecer dúvidas de pacientes e cuidadores, além de estabelecer vínculo entre profissional-paciente-família.

\section{CONCLUSÃO}

Constatou-se que as condições periodontais e de higiene bucal dos pacientes com necessidades especiais incluídos neste estudo são insatisfatórias, visto que foram encontrados quadros de alto índice de pacientes com placa e sangramento gengival espontâneo. Dessa forma, ações preventivas em saúde bucal focadas em orientação aos pacientes e cuidadores são fundamentais para a efetividade da higiene bucal.

\section{REFERENNCIAS}

1. Sacchetto MSLS, Andrade NS, Brito MHSF, Lira DMMP, Barros SLLV. Evaluation of oral health in patients with mental disorders attended at the clinic of oral diagnosis of a public university. Rev Odontol UNESP. 2013 Set-Out;42(5):344-9. http://dx.doi.org/10.1590/S180725772013000500005.

2. Gardens SJ, Krishna M, Vellappally S, Alzoman H, Halawany HS, Abraham NB, et al. Oral health survey of 6-12-year-old children with disabilities attending special schools in Chennai, India. Int J Paediatr Dent. 2014 Nov;24(6):424-33. http://dx.doi.org/10.1111/ipd.12088. PMid:24372860

3. Moursi AM, Fernandez JB, Daronch M, Zee L, Jones CL. Nutrition and oral health considerations in children with special health care needs: implications for oral health care providers. Pediatr Dent. 2010 Jul-Ago;32(4):333-42. PMid:20836954.

4. Oliva L. Oral hygiene in the special needs classroom. NASN Sch Nurse. 2013 Nov;28(6):281-3. http://dx.doi.org/10.1177/1942602X13506751. PMid:24392505

5. Lewis CW. Dental care and children with special health care needs: a population-based perspective. Acad Pediatr. 2009 Nov-Dez;9(6):420-6. http://dx.doi.org/10.1016/j.acap.2009.09.005. PMid:19945077

6. Campos CC, Frazão BB, Saddi GL, Morais LA, Ferreira MG, Setúbal PCO, et al. Manual prático para o atendimento odontológico de pacientes com necessidades especiais. 2. ed. Goiânia: Universidade Federal de Goiânia; 2009.

7. Avenali L, Guerra F, Cipriano L, Corridore D, Ottolenghi L. Disabled patients and oral health in Rome, Italy: long-term evaluation of educational initiatives. Ann Stomatol (Roma). 2011 Mar;2(3-4):25-30. PMid:22545186.

8. Carvalho EMC, Araújo RPC. A saúde bucal em portadores de transtornos mentais e comportamentais. Pesq Bras Odontoped Clin Integr. 2004 Jan-Abr;4(1):65-75.

9. Uemura ST, Ramos L, Esposito D, Uemura AS, Boccia MF, Mugayar LRF. Motivação e educação odontológica em pacientes especial. RGO - Rev Gaúcha Odontol. 2004 Abr-Jun;52(2):91-100. 
10. Campanaro M, Huebner CE, Davis BE. Facilitators and barriers to twice daily tooth brushing among children with special health care needs. Spec Care Dentist. 2014 Jul-Ago;34(4):185-92. http://dx.doi.org/10.1111/scd.12057. PMid:24252060

11. Nahar SG, Hossain MA, Howlader MB, Ahmed A. Oral health status of disabled children. Bangladesh Med Res Counc Bull. 2010 Ago;36(2):613. PMid:21473203.

12. Löe H, Silness J. Periodontal disease in pregnancy.I. Prevalence and severity. Acta Odontol Scand. 1963 Dez;21(6):533-51. http://dx.doi. org/10.3109/00016356309011240. PMid:14121956

13. Löe H. The gingival index, the plaque index and the retention index system. J Periodontol. 1967 Nov-Dez;38(6):610-6. http://dx.doi. org/10.1902/jop.1967.38.6.610. PMid:5237684

14. Ameer N, Palaparthi R, Neerudu M, Palakuru SK, Singam HR, Durvasula S. Oral hygiene and periodontal status of teenagers with special needs in the district of Nalgonda, India. J Indian Soc Periodontol. 2012 Jul;16(3):421-5. http://dx.doi.org/10.4103/0972-124X.100923. PMid:23162340

15. Trentin MS, Silva SO, Linden MSS. Prevalence of periodontal disease in special needs patients at APAE-PF/RS and the effect of local prevention programs. Braz J Oral Sci. 2010; 9(4):475-80.

16. Menchaca MHR, Alanis TMG, Silva RG. Guía para el cuidado de la salud oral en pacientes con necesidad de cuidados especiales de salud en México. Rev ADM. 2011; 68(5):222-8.

17. Bhandary S, Shetty V, Hegde AM, Rai K. Knowledge of care providers regarding the oral health care of visually impaired children. J Clin Pediatr Dent. 2013; 37(4):385-9. PMid:24046987.

18. Ahmad MS, Jindal MK, Khan S, Hashmi SH. Oral health knowledge, oral hygiene status and dental caries prevalence among visually impaired students in residential institute of Aligarh. J Dent Oral Hygiene. 2009; 2(1):22-6.

19. Stiefel DJ. Dental care considerations for disabled adults. Spec Care Dentist. 2002; 22(3 Suppl):26S-39S. PMid:12375745.

20. Blevins JY. Oral health care for hospitalized children. Pediatr Nurs. 2011 Set-Out;37(5):229-35, quiz 236. PMid:22132567.

21. Fisher K. Is there anything to smile about? A review of oral care for individuals with intellectual and developmental disabilities. Nurs Res Pract. 2012; 2012:860692. PMid:22548164.

22. Silva APR, Gomide MR. Análise da higiene oral, ocorrência de cáries e padrões alimentares de um grupo de pacientes especiais. J Bras Clín Odontol Integr Saúde Bucal Coletiva. 2006;1-5.

23. Cericato GO, Fernandes APS. Implicações da deficiência visual na capacidade de controle de placa bacteriana e perda dentária. RFO. 2008; 13(2):17-21.

24. Carvalho EMC, Araujo RPC, Correa AP. Perfil periodontal de portadores de transtorno mental e comportamental assistidos no Hospital Juliano Moreira - Bahia. Rev Fac Odontol Univ Fed Bahia. 2001; 22(1):26-44.

25. Avasthi K, Bansal K, Mittal M, Marwaha M. Oral health status of sensory impaired children in Delhi and Gurgaon. International Journal of Dental Clinics. 2011; 3(2):21-3.

26. Azrina AN, Norzuliza G, Saub R. Oral hygiene practices among the visually impaired adolescents. Annal Dent Univ Malaya. 2007; 14:1-6.

27. Peixoto ITA, Rocha CT, Fernandes PM. Auxiliary devices for management of special needs patients during in-office dental treatment or at-home oral care. Int J Dent. 2010; 9(2):85-9.

28. Ferguson FS, Cinotti D. Home oral health practice: the foundation for desensitization and dental care for special needs. Dent Clin North Am. 2009 Abr;53(2):375-87, xi. http://dx.doi.org/10.1016/j.cden.2008.12.009. PMid:19269405

29. Aguiar SMHCA, Alves Rezende MCR. Dental care for cerebral palsy patients using auxiliary resources to inhibit pathological tonic reflexes. Arch Health Invest. 2013; 2(4):39-44.

30. Parizotto SPCOL, Ruschel HC, Rodrigues CRD, Mosele GTN. Eficácia de duas técnicas de utilização de fio dental e sua associação com a ocorrência de traumatismo gengival em crianças. Pesqui Bras Odontopediatria Clín Integr. 2009; 9(1):19-23. http://dx.doi.org/10.4034/15 19.0501.2009.0091.0004.

31. Al-Shammari KF, Al-Ansari JM, Al-Khabbaz AK, Dashti A, Honkala EJ. Self-reported oral hygiene habits and oral health problems of Kuwaiti adults. Med Princ Pract. 2007; 16(1):15-21. http://dx.doi.org/10.1159/000096134. PMid:17159358

32. Shyama M, Honkala S, Al-Mutawa SA, Honkala E. Oral health-related quality of life among parents and teachers of disabled schoolchildren in Kuwait. Med Princ Pract. 2013; 22(3):285-90. http://dx.doi.org/10.1159/000345213. PMid:23171756

\section{CONFLITOS DE INTERESSE}

Os autores declaram não haver conflitos de interesse.

\section{*AUTOR PARA CORRESPONDÊNCIA}

Lisandrea Rocha Schardosim, Rua Gonçalves Chaves, 457, Centro, 96015-560 Pelotas - RS, Brasil, e-mail: lisandrears@hotmail.com 